\title{
Clusters and Nuclear Physics - A Prolific Union
}

The study of atomic and molecular clusters is developing at a rapid - at times even tumultuous - pace. Among the most conspicuous discoveries are the $\mathrm{C}_{60}$ molecule, the buckyball, and the associated family of analogous molecules, the fullerenes [1]. It was also quite unexpected that atoms as simple as the noble gases would cluster into specific "magic shell" configurations, as quasi-crystals of the Mackay icosahedral type [2]; and the magic continued with the discovery of electronic shell structure in clusters of sodium [3]. These and many other observations are now challenging our understanding of how matter organizes itself on the atomic scale. Good models with predictive power are in heavy demand. In this situation it turns out that nuclear physics can be a source of inspiration and guidance, in particular with respect to substances that have the character of Fermi liquids. Atomic nuclei are themselves clusters of the constituent protons and neutrons; and this particular type of cluster has been very thoroughly studied during more than 60 years.

The nucleus is clearly a quantum liquid: it does not crystallize, and it behaves in fusion and fission reactions like a liquid drop, while at the same time exhibiting pronounced quantum features near the ground state. The most conspicuous ones are the shell structure and symmetry breaking in the form of non-spherical shapes.

\section{Shell Structure and Deformation in Fermi Liquids}

Among the newcomers to the quantum liquid family, cold droplets of ${ }^{3} \mathrm{He}$ are the closest relatives to nuclei. Unfortunately, experiments with millikelvin, electrically neutral ${ }^{3} \mathrm{He}$ clusters are exceedingly difficult [4]. The system of conduction electrons in clusters of simple metals $(\mathrm{Li}, \mathrm{Na}, \mathrm{K}, \mathrm{Rb}, \mathrm{Cs}, \mathrm{Cu}$, $\mathrm{Ag}$, and $\mathrm{Au}$ ) is much more readily accessible to experiment. In a metal, the electrons co-exist with the positive metal ions, together forming a medium of constant density. It turns out - as could also be expected after a little reflection - that the specific quantum properties of small metal clusters

Professor S. Bjørnholm has worked since 1955 at The Niels Bohr Institute, University of Copenhagen, Blegdamsvej 17, DK-2100 Copenhagen $\varnothing$. After graduating in chemistry from the Technical University of Denmark in 1953, he started his research career at the Pasteur Institute in Paris with work on the physical structure of proteins. His main interests from 1955-86 have been the structure and dynamics of atomic nuclei, with a brief excursion in 1973-76 into energy planning. The commitment to cluster research emerged after he became aware in 1985 of analogies between the quantum structure of metal clusters and of nuclei and helium.

\section{S. Bjørnholm}

\author{
The Niels Bohr Institute, University of Copenhagen
}

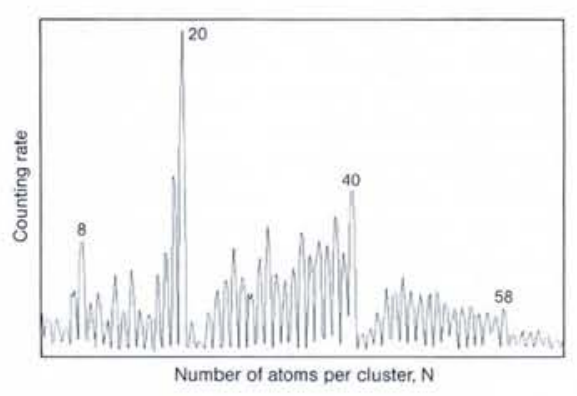

Fig. 1 - Abundance spectrum of sodium clusters according to the number $N$ of atoms per droplet. Magic numbers 8, 20, 40, and 58 appear as maxima, followed by a pronounced minimum. (From [3]).

are completely dominated by the system of delocalized conduction electrons. To a good approximation they form a Fermi liquid, like the nucleus.

In a now celebrated experiment by W.D. Knight et al. at Berkeley in 1984 [3], the analogy to nuclei was strikingly demonstrated. It was a (seemingly) quite simple experiment. Sodium vapour from an oven, pressurised with excess argon, was allowed to expand into a vacuum through a supersonic nozzle. As with steam from a tea kettle, a stream of clusters was produced and the experiment simply consisted in measuring the frequency of the occurrence of sodium clusters of different sizes. The resulting mass spectrum, Fig. 1, shows pronounced peaks at what appeared to begin with to be "magic" numbers. After consulting a classic text in nuclear physics [5] it was soon realized that $2,8,20,40,58$, etc. are the positions of closed shells for independent fermions moving in a spherical potential well, with a potential that is uniform across most of the inside of the sphere and rises steeply on approaching the surface.

This was the first rendezvous of nuclear physics with cluster science. But the Berkeley group went one step further. Scrutinizing the spectrum in Fig. 1 more closely they wondered about the missing signature expected from a sub-shell closing within the major shell $N=20-40$ (that contains two different $l$-states). Again nuclear physics could produce the likely answer. Closed shell nuclei are spherical, but open-shell nuclei tend to be deformed (they have ellipsoidal shapes). As a result, subshell degeneracies are lifted. This is described by the Nilsson-Strutinsky model in nuclear physics. With a small modification (deletion of the strong spin-orbit term), this model is now currently known to cluster physicists as the Cleminger-Nilsson model [6]. To first order, it is quite successful in explaining the observed consequences of symmetry breaking in clusters of simple metals.

\section{Supershells}

The inspiration also goes the other way, of course. Nuclear physicists have been deeply absorbed in the question of how the nuclear shell structure extrapolates to nuclei with hundreds, even thousands, of nucleons. Through such extrapolations they are first of all able to predict islands of stability of closed shell nuclei beyond the presently known table of isotopes. But the theoretical studies are also addressing the general question of the correspondence between quantum shell structure and ordered classical motion in the limit of very large quantum numbers. Here cluster experiments produce an answer beyond any realistic possibilities within nuclear physics itself.

Again, the experiment [7] is conceptually quite simple. It amounts to an extension of the Knight et al. cluster production technique with size analysis, Fig. 1 , to larger sizes. In this way it has been possible to identify shell closings at specific magic numbers $N_{0}$ all the way up to $N=3000$. So far one has observed altogether 22 shells and consequently measured 22 values of $N_{0}$. The spacings $\Delta N_{0}$ and the amplitudes of the measured shell effect vary in a regular fashion from one shell to the next. About five strong shells are followed by weaker shells as the cluster grows in size. A set of stronger shells then follows, and so on. Where the shell structure is weak there are also irregularities in the spacings between the shells. All this can be illustrated by expressing the magic numbers in terms of the corresponding cluster radii

$$
R_{0}=r_{\text {WS }} N_{0}{ }^{1 / 3}
$$

where $r_{W S}$ is the radius in the sphere available to each atom in the metallic medium (the Wigner-Seitz radius) and drawing circles corresponding to the experimentally measured magic-drop radii $R_{0}$. The result is sketched in the lower part of Fig. 2, and can be thought of as a picture of cuts through the specially selected "magic" cluster droplets, only on the nanometre scale. On the average, the radius increments are equal to $0.61 r_{\text {ws }}$, and one sees a beating pattern. This pattern can be described in terms of a superposition of two sets of strictly equidistant sets of concentric spheres with radius increments of $0.58 r_{W S}$ 


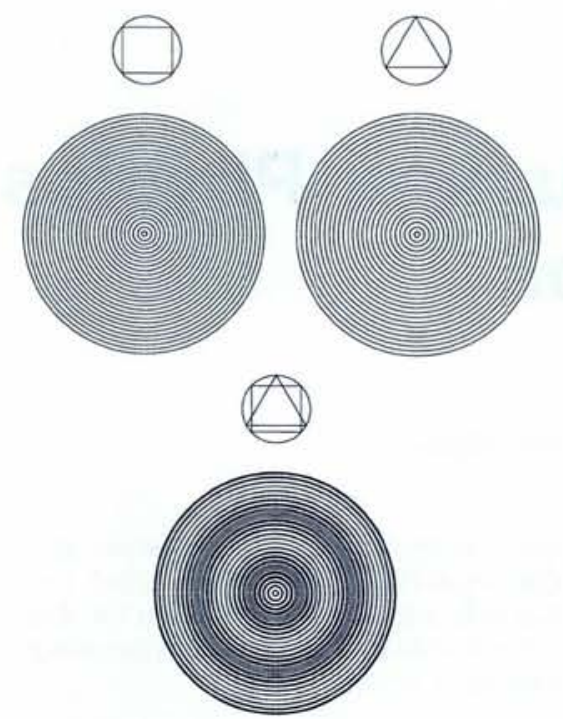

and $0.64 r_{\text {WS }}$, respectively (Fig. 2, upper part). Miraculously, if one multiplies the length $L$ of a square inscribed in the first set of these circles, $L=4 \sqrt{2} R_{0}$, with the Fermi momentum $p_{\mathrm{F}}$ of a metallic electron

$$
p_{F}=(\pi / 4)^{1 / 3}\left(h / r_{W S}\right)
$$

one obtains action values $p_{\mathrm{F}} L$ that increase by one unit of Planck's constant with each circle. The same also happens for triangles inscribed in the second set of circles $\left(L=3 \sqrt{3} R_{0}\right)$.

This purely experimental observation may evoke associations to Bohr's first model of the hydrogen atom and his explanation of Balmer's formula for the spectral lines of hydrogen. Are the triangles and squares analogous to Bohr's quantized Kepler ellipses? In a spherical potential well that is uniform inside, the classical trajectories are indeed straight lines so a closed classical orbit will be a polygon. It could be that the electronic eigenstates in the cluster are simply quantized triangles and squares. In that case, what about quantized pentagons, hexagons, or starlike orbits inside the metal sphere? They should also give rise to specific eigenstates. These are questions nuclear physicists have addressed long ago [8]. The hydrogen inverse radius potential and its eigenvalue spectrum is a particularly simple case. Here it is possible to associate each quantum state with a definite closed classical orbit, in a one-to-one correspondence. Consequently, individual spectral lines are described in terms of two quantized ellipses. For other radial, but still spherically symmetric, potentials the situation is not so simple. One can analyze individual quantum states in terms of closed classical orbits in the semiclassical theory [8], but this requires an infinite set of closed orbits for each quantum state. There is no one-to-one correspondence. If one truncates the complete set of orbits at some point, retaining only the shorter orbits, one still obtains a good description of the density of single particle states as a function of energy, or alternatively, the density of states at the Fermi energy as a function of particle number. The cluster experiment is a reflection of just that quantity; and the semi-classical theory predicts shell modulations of precisely the kind implied by experiment. This again reflects
Fig. 2 - Experimental magic numbers $N_{O}$, converted to magic radii $R_{0}$, according to the equation $R_{O}=r_{W S} N_{O}^{1 / 3}$, for sodium with up to 3000 atoms per cluster. The beat pattern which is seen in the experimental results and in the lower part of the figure, can be reproduced by superposing the two sets of concentric rings shown in the upper part of the figure. (Based on [7]).

the fact that in the theory it is the two shortest orbits, triangles and squares, which are playing the most important rôle in shaping the level-density modulations.

Nuclear and cluster theorists have joined to make more detailed semi-classical studies of the supershells [9]. Replacing the original square-well potentials with more realistic ones of the Woods-Saxon type, with parameters appropriate to sodium droplets, one obtains quantitative agreement with experiment. (The shell and supershell features are also contained in the eigenvalue spectrum obtained by numerical solution of the Schrödinger equation for the Woods-Saxon potential, but the physical interpretation is not so clear in this approach [9].)

\section{Giant Resonances and Plasmons}

One aspect of shell structure is the spontaneous breaking of the spherical symmetry for Fermi droplets with a partly filled, highly degenerate state [8]. To some it is the familiar Jahn-Teller effect. As mentioned already, it is well known that non-magic nuclei tend to be spheroidally deformed and that one expects the same for metal clusters [6]. The experimental demonstration of this is not straightforward since the standard methods from nuclear physics, such as spectroscopic identification of rotational bands or the identification of large electric quadrupole moments, are not easily applied in cluster experiments. On the other hand - as the Berkeley group once again realized - the splitting of the nuclear giant dipole resonance has its analogue in clusters [10]. In nuclei, gamma rays of the right frequency will excite a collective state where the protons oscillate jointly against the neutrons. This rather broad state exhausts almos
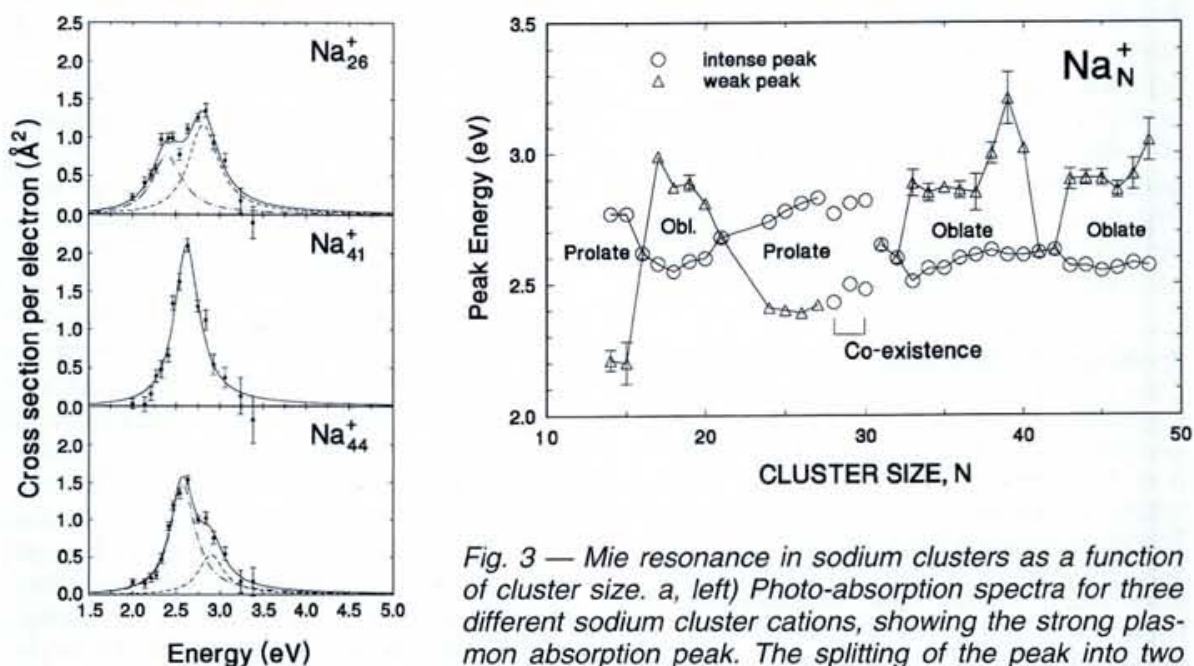

Fig. 3 - Mie resonance in sodium clusters as a function of cluster size. a, left) Photo-absorption spectra for three different sodium cluster cations, showing the strong plasmon absorption peak. The splitting of the peak into two components is interpreted as a sign of non-sphericity of the clusters. b, right) The occurrence of prolate or oblate deformations in sodium cluster cations, as measured using the plasmon peak profiles of a). It is seen that the shape of the cluster varies systematically. (From [11]). the entire dipole sum rule. If the nucleus is axially deformed the resonance splits up into two states with a frequency ratio equal to the ratio of the two axes of the spheroid, providing in this way a measure of the deformation. For an axially symmetric prolate deformation, the doubly degenerate highfrequency peak will be twice as intense as he singly degenerate low-frequency peak. clusters, called the Mie plasmon, where the valence electrons oscillate collectively relative to the metal ions. It also splits into two (or three) peaks if the cluster is deformed, the frequency splitting in fact being $3 / 5$ of what you would expect for nuclei. (The restoring forces responsible for the oscillations are after all very different in the two Fermi liquids). With inspiration from nuclear physics, several groups interested in clusmetal clusters as a function of size $\mathrm{N}$. Fig. $3 a$ is an example from work at my own institute [11]. It is part of systematic study bracketing the two shell closures at electron numbers 20 and 40 , respectively (the relevant cluster cations will then have mass 21 and 41, respectively). In the open-shell clusters, e.g., $\mathrm{Na}_{26}^{+}$and $\mathrm{Na}_{44}^{+}$, the
resonance is clearly seen to be split into (at least) two peaks, reflecting deformation, while $\mathrm{Na}_{41}^{+}$shows a single peak as expected for a spherical closed shell cluster. In $\mathrm{Na}_{26}^{+}$, the high-energy peak is the more intense, indicating prolate deformation, while the peak profile in $\mathrm{Na}_{44}^{+}$indicates oblate deformation. The occurrence of oblate or prolate varies systematically. Fig. $3 b$, where all the measured peak positions are plotted. The Cleminger-Nilsson model, or similar models based, for example, on deformed Woods-Saxon potentials, predict the same general trends [12].

These experiments illustrate very nicely how the same underlying physics is expressed in both nuclei and metal clusters. The cluster experiments may potentially add considerably to what is already known about deformations from nuclei, and perhaps future experiments will do so. It is again a 
question of going beyond the limit that Nature has imposed for the maximum size of nuclei. The relative occurrence of prolate versus oblate shapes is a non-trivial issue that has been discussed a good deal in nuclear physics. Semi-classical theory offers a rather transparent model description of this in terms of the response of triangular and other closed classical orbits to deformation, but the problem is that one needs larger Fermi droplets than nuclear physics can provide to test the theory.

\section{Fission}

The break-up of a drop of quantum liquid under the influence of its electric charge, i.e., fission, can be studied from a new angle by examining the fission of multiply charged metallic clusters. In all cases the fission process depends on the competition between the cohesive surface force and the repulsive electrical force. The relevant parameter is therefore the ratio of the Coulomb energy to (two times) the surface energy of a sphere, i.e.,

$$
E_{\mathrm{c}} / 2 E_{\text {surf }}=\left(Q^{2} / N\right) /\left(Q^{2} / N\right)_{\text {crit }}
$$

where $Q$ is the charge. Inserting typical material constants into the equation one realizes that while 200 nucleons can hold 90 elementary charges together, the same number of metal atoms can hold at most 9 excess charges without immediately fissioning. This reflects the difference between nuclear cohesion, which is due to the strong force, and metallic cohesion which is of electromagnetic origin.

A new stage is set for fission studies thanks to this difference. Quantum effects are likely to be much more pronounced in metallic clusters than in nuclei (modifications of the smooth liquid-drop picture owing to quantum effects at the fission-like saddlepoint shape makes fission interesting already in nuclei). There are some intriguing experimental results for the fission of doubly charged silver and potassium clusters [13] with sizes up to $N=30$. The division into different fragment pairs seems indeed to be dominated by quantum effects for symmetric mass splits. For clusters in this size range one expects the saddle shapes to be (nearly) two spheres in contact, so the quantum effects are those relating to spherical shapes. Since these shapes can be studied by other means, it would be even more interesting to investigate, using new experiments, the fission of larger clusters with charges greater than 2 since they can have saddle shapes that do not resemble two spheres in contact.

\section{Atypical Molecules: \\ A Bridge to Chemistry}

The cooling of a hot metallic droplet through evaporation of (neutral) atoms has much in common with neutron evaporation from the compound nucleus. In both cases the process is described by transition-state theory. The difference lies in the level-density laws. For nuclei, the level-density law of a fermion system alone applies, while for a metal cluster the level density is a product of the ionic, i.e., the oscillator level density, and the fermionic electron level densities. It is the latter that is strongly modulated by the cluster's shell structure, so the fermionic structure plays an important rôle in evaporation processes involving clusters, and in describing how shell structure becomes washed out with increasing temperature. There exists here a bridge to transition-state theory in chemistry, where the emphasis lies on the oscillator level density, while the electrons are assumed not to contribute because they presumably remain in their adiabatic ground state during typical molecular dissociation processes. In this sense, metal clusters are atypical molecules. Once again we see how a marriage between the young science of cluster physics and a more mature partner can be rich in mutual inspiration.

[1] Curl R.F. and Smalley R.E., Scientific American 265 (1991) 32.

[2] Echt O., Sattler K. and Recknagel E., Phys. Rev. Lett. 47 (1981) 1121.

[3] Knight W.D. et al., Phys. Rev. Lett. 52 (1984) 2141.

[4] Buck U., Europhysics News 20 (1989) 41. [5] Mayer M.G. and Jensen J.H.D., Elementary Theory of Nuclear Shell Structure (Wiley, New York) 1955.

[6] Clemenger K., Phys. Rev. B 32 (1985) 1359.

[7] Pedersen J. et al., Nature 353 (1991) 733. [8] Balian R. and Bloch C., Ann. Phys. 69 (1971) 76; Bohr A. and Mottelson B.R., Nuclear Structure, Vol. 11 (Benjamin, London, 1975) p. 578.

[9] Nishioka H., Hansen K. and Mottelson B.R., Phys. Rev. B 42 (1990) 9377; Brack M., Genzken 0. and Hansen K., Z. Phys. D 21 (1991) 65

[10] Selby K. et al., Phys. Rev. B 40 (1989) 5417.

[11] Pedersen J. et al., Z. Phys. D 26 (1993) 281.

[12] Frauendorf S. and Pashkevich V.V., $Z$. Phys. D 26 (1993) S98.

[13] Katakuse I., Ito H. and Ichihara T., Int. J. Mass Spectrometry \& Ion Processes 97 (1990) 47; Brechignac C. et al., Phys. Rev. B 47 (1991) 11386.

\section{HEWLETT-PACKARD EUROPHYSICS PRIZE} New Molecular Forms of Carbon

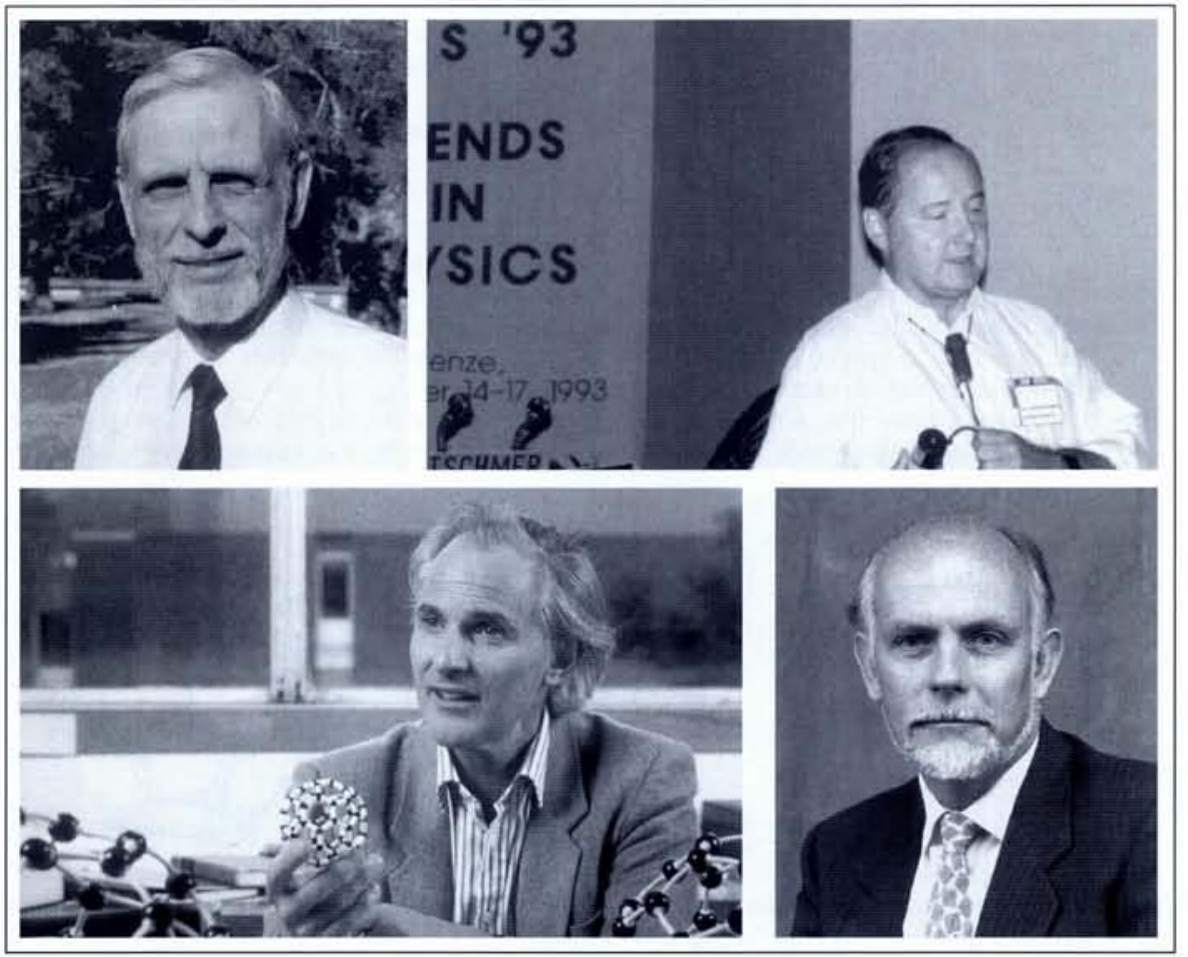

The 1994 Hewlett-Packard Europhysics Prize of EPS for outstanding achievements in condensed matter physics has been awarded for "the discovery of new molecular forms of carbon and their production in the solid state" to D.R. Huffman (The University of Arizona, Tucson, USA), W. Krätschmer (Max-Planck Institut für Kernphysik, Heidelberg, Germany), H. Kroto (University of Sussex, UK), and R.E. Smalley (Rice University, Houston, USA).

Donald Huffman was honoured last year for the discovery of $\mathrm{C}_{60}$ by being appointed the Regents' Professor of Physics in the Department of Physics, The University of Arizona, Tucson, Arizona, USA. He studied at Texas A\&M University and at Rice University before receiving his Ph.D. from the University of California, Riverside, in 1966. After working as a National Science Foundation postdoc at the University of Frankfurt, he joined The University of Arizona as an Assistant Professor in 1968, becoming Professor in 1975. Professor Huffman, whose research focuses on the optical properties of small particles, has visited Europe regularly, first as a Visiting Scholar to the University College, Cardiff, and then on a sabbatical to the European Space Agency operations in Noodwijk and as

Clockwise from the upper left: D.R. Huffman, W. Krätschmer, R.E. Smalley, and H. Kroto. 